\title{
UTILIZAÇÃO DE DIFERENTES EMBALAGENS PLÁSTICAS PARA A CONSERVAÇÃO DE PRODUTO MINIMAMENTE PROCESSADO DE MANGAS 'TOMMY ATKINS'1
}

\author{
LEANDRO CAMARGO NEVES ${ }^{2}$, VANUZA XAVIER DA SILVA ${ }^{3}$, \\ LUCILÉIA RODRIGUES FERRAZ ${ }^{3}$, MARCOS ANDRÉ DE SOUZA PRILL ${ }^{4}$ \\ SERGIO RUFFO ROBERTO 5
}

\begin{abstract}
RESUMO-O trabalho objetivou a avaliação do potencial de conservação de mangas 'Tommy Atkins', minimamente processadas, utilizando-se de diferentes tipos de embalagens plásticas para o estabelecimento da atmosfera modificada (AM) passiva. Os frutos, colhidos fisiologicamente maturos, apresentavam coloração roxo-esverdeada (1:1), teor de sólidos solúveis (SS) 8,9 $\pm 0,3^{\circ} \mathrm{Brix}$, acidez titulável (AT) de $1,25 \pm 0,5 \mathrm{~g}$ de ácido cítrico. $100 \mathrm{~g}$ de polpa ${ }^{-1}$ e massa fresca de $565 \pm 25 \mathrm{~g}$. Os frutos, após a higienização em solução previamente acidificada $(\mathrm{pH}=3,0)$ de hipoclorito de sódio $(\mathrm{NaOCl})$, a $100 \mathrm{mg} \cdot \mathrm{L}^{-1}$, por 10 minutos, e seccionados em pedaços de $0,02 \pm 0,01 \mathrm{~m}$ de largura e $0,05 \pm 0,015 \mathrm{~m}$ de comprimento, foram novamente higienizados em solução previamente acidificada de $\mathrm{NaOCl}(\mathrm{pH}=3,0)$, a $10 \mathrm{mg} \cdot \mathrm{L}^{-1}$, por 3 minutos. Os pedaços, no formato de cubos de $0,02 \pm 0,01 \mathrm{~m}$ de largura e $0,05 \pm 0,015 \mathrm{~m}$ de comprimento, foram acondicionados em bandejas rígidas de poliestireno $(0,19 \times 0,12 \mathrm{~m})$, com capacidade para $250 \mathrm{~g}$ e revestidas com os seguintes materiais: T1 (controle): filme perfurado de polietileno de baixa densidade (PEBD) de $0,006 \mathrm{~mm}$ ( 35 perfurações na superfície da embalagem, simulando a atmosfera convencional); T2: filme de PEBD de 0,020 mm; T3: filme de PEBD de 0,060mm; T4: filme de PEBD de 0,080mm; T5: filme de polipropileno (PP) de 0,052mm, e T6: bandeja rígida de polietileno tereftalato (PET), herméticas, com capacidade para $500 \mathrm{~mL}$ e dimensões de $0,22 \times 0,12 \times 0,11 \mathrm{~m}$, com tampa do mesmo material, sem perfurações e sem o revestimento plástico. Os tratamentos foram acondicionados em câmara frigorífica a $10 \pm 0,5^{\circ} \mathrm{C}$ e $90 \pm 3 \%$ de U.R, por 10 dias. Não foi constatada diferença significativa entre os tratamentos para as análises de SS e AT. A maior atividade microbiológica foi observada nos frutos embalados em filmes de PEBD de 0,006 e $0,020 \mathrm{~mm}$. As menores perdas de massa fresca, a melhor manutenção dos teores de ácido ascórbico, a ausência de contaminação microbiana, o melhor aspecto visual e a preferência dos julgadores na análise sensorial foram atribuídos aos frutos acondicionados nas embalagens PET.
\end{abstract}

Termos para indexação: Vida útil, atmosfera modificada, Mangifera indica L.

\section{DIFFERENT PLASTIC PACKING FOR PRODUCT CONSERVATION OF MANGOES 'Tommy Atkins' MINIMALLY PROCESSED}

\begin{abstract}
The aim of this work was to evaluate the conservation potential of 'Tommy Atkins' mangoes minimally processed by using different types of plastic cover to establish a passive modified atmosphere (MA). The fruits, collected in mature-green stage, showed purple-green colour (1:1), SS content of $8.9 \pm 0.3$ ${ }^{\circ}$ Brix, titratable acidity (TA) of $1.25 \pm 0.5 \mathrm{~g}$ of citric acid. $100 \mathrm{~g}$ flesh $^{-1}$ and fresh mass of $565 \pm 25 \mathrm{~g}$. The fruits, after sterilized in an acid solution $(\mathrm{pH}=3.0)$ of chlorine $(\mathrm{NaOCl}), 100 \mathrm{mg} \cdot \mathrm{L}^{-1}$, during 10 minutes, were chopped into parts of $0.02 \pm 0.01 \mathrm{~m}$ of width and $0.05 \pm 0.015 \mathrm{~m}$ of length, one more time sterilized in an acid solution $(\mathrm{pH}=3.0)$ of chlorine $(\mathrm{NaOCl}), 10 \mathrm{mg} \cdot \mathrm{L}^{-1}$, during 3 minutes. The parts, in a cube format of $0.02 \pm 0.01 \mathrm{~m}$ width and $0.05 \pm 0.015 \mathrm{~m}$ length, were placed in rigid poliestiren trays $(0.19 \mathrm{x} 0.12 \mathrm{~m})$, with capacity for $250 \mathrm{~g}$ and covered with the following materials: T1 (control): low density perfured polyetilen film (LDPPF) of $0.006 \mathrm{~mm}$ ( 35 perforations in the surface of the packing to simulate the conventional atmosphere); T2: LDPE film of $0.020 \mathrm{~mm}$; T3: LDPE film of $0.060 \mathrm{~mm}$; T4: LDPE film of $0.080 \mathrm{~mm}$; T5: polypropylene film (PP) of $0.052 \mathrm{~mm}$ and T6: hermetic rigid polietilen tereftalate (PET), with capacity for $500 \mathrm{~mL}$ and dimensions of $0.22 \times 0.12 \times 0.11 \mathrm{~m}$, with lid cover of the same material, without perforations and without the plastic cover. The treatments were placed in a cold chamber at $-10 \pm 0.5^{\circ} \mathrm{C}$ and $90 \pm 3.0 \%$ of R.U. for 10 days. It was not observed significative difference among treatments for SS and TA analysis. The higher microbilogical activity was observed in fruits covered with LDPPF films of 0.006 and LDPE of $0.20 \mathrm{~mm}$. The lower loss of fresh matter, the best maintenance of ascorbic acid, the absence of microbiological contamination, the best visual aspect and the panelists' preference in the sensorial analysis were observed in fruits placed in PET containers.
\end{abstract}

Index terms: shelf-life, modified atmosphere, Mangifera indica L.

\footnotetext{
1(Trabalho 239-08). Recebido em: 17-09-2008). Aceito para publicação em: 13-01-2009.

${ }^{2}$ UFRR - Prof.. Dr. Depto de Fitotecnia - CCA/UFRR, Km 12 BR174 s/nº, CEP 69301-970, Boa Vista-RR. rapelbtu@hotmail.com ${ }^{3}$ UFRR - Graduanda em Agronomia - CCA/UFRR, Km 12 BR174 s/nº, CEP 69301-970, Boa Vista-RR. rmbenedette@hotmail.com ${ }^{4}$ UFRR - Eng Agr. Esp. Ciência e Tec. de Alimentos - CCA/UFRR, Km 12 BR174 s/nº CEP 69301-970, Boa Vista-RR. marcosprill@bol.com.br ${ }^{5}$ UEL - Prof. Dr. Depto Agronomia - Centro de Ciências Agrárias, C.P. 6001, CEP 86051-990, Londrina-PR. sroberto@uel.br
} 


\section{INTRODUÇÃO}

Uma alternativa aos desperdícios de frutos, em média de 20 a $30 \%$ em alguns dos grandes centros de distribuição (Junqueira \& Luengo, 2000), seria o processamento mínimo de frutos (Kluge et al., 2003). Essa tecnologia, também chamada de "fresh cut", permite a obtenção de produtos frescos, convenientes e diferenciados, possibilitando, dentre outros fatores, a agregação de valor ao produto final.

Entretanto, muitos seriam os condicionantes para a efetivação desta tecnologia como ferramenta prática e usual ao agroempresário. O controle microbiológico (Verlinden \& Nicolai, 2000) e o controle da aceleração dos processos pertinentes ao avanço da senescência dos frutos (Kluge et al., 2003; Ahvenainen, 1996), principalmente durante as etapas de seccionamento, descascamento e acondicionamento (Cantwell \& Suslow, 2002), poderiam comprometer a execução e o sucesso do processamento mínimo de frutos.

Apesar da praticidade, conveniência e a provável potencialização mercadológica, o processamento mínimo pode provocar nos frutos e hortaliças comportamento similar a de tecidos submetidos a algum tipo de estresse (Hong \& Kim, 2001), que pode conduzir alterações bioquímicas e fisiológicas indesejáveis. Sendo assim, existe a necessidade da utilização da combinação de processamentos brandos, como, por exemplo: a desinfecção com soluções de hipoclorito de sódio ou sódio ativo (Pereira et al., 2003; Arruda et al., 2003), o uso de soluções de $\mathrm{CaCl}_{2}$ (Antoniolli et al, 2000); e a utilização de AM, pelo uso de embalagens plásticos (Pereira et al., 2003; Arruda et al., 2003); desse modo, procura-se manter a qualidade sensorial e microbiológica do produto minimamente processado.

Neste sentido, o objetivo deste trabalho foi avaliar a interação do armazenamento refrigerado (AR) de mangas 'Tommy Atkins', minimamente processadas, associado a diferentes tipos de embalagens plásticas com efeito de AM passiva.

\section{MATERIAL E MÉTODOS}

Utilizou-se de mangas 'Tommy Atkins' obtidas em propriedade agrícola localizada na zona rural de Boa Vista-RR, situada na latitude 2 ${ }^{\circ} 50^{\prime} 06^{\prime \prime}$ $\mathrm{N}$ e longitude $60^{\circ} 40^{\prime} 28^{\prime \prime} \mathrm{W}$, e transportados até o Laboratório de Tecnologia de Alimentos/UFRR (15 $\pm 2^{\circ} \mathrm{C}$ e $75 \pm 3 \%$ de U.R.) em contentores de $20 \mathrm{~kg}$, onde foram selecionados pela ausência de danos e/ ou podridões e padronizados segundo os atributos visuais (coloração e tamanho).
Colhidas fisiologicamente maturas, as mangas 'Tommy Atkins' apresentavam coloração roxoesverdeada (1:1), teor de SS 8,9 $\pm 0,3^{\circ}$ Brix, AT de $1,25 \pm 0,5 \mathrm{~g}$ de ácido cítrico. $100 \mathrm{~g}$ de polpa ${ }^{-1}$ e massa fresca de $565 \pm 25 \mathrm{~g}$. Antes da embalagem, conforme recomendado por Elesbão et al. (2006), os frutos foram higienizados em solução previamente acidificada $(\mathrm{pH}=3,0)$ de hipoclorito de sódio $(\mathrm{NaOCl})$, a 100 mg. $\mathrm{L}^{-1}$, e seccionados em cubos de $0,02 \pm 0,01 \mathrm{~m}$ de largura e $0,05 \pm 0,015 \mathrm{~m}$ de comprimento. Após, esses pedaços foram novamente higienizados, em solução previamente acidificada $(\mathrm{pH}=3,0)$ de $\mathrm{NaOCl}$, a 10 mg. $\mathrm{L}^{-1}$, por 3 minutos. Realizado o enxágue e a secagem dos pedaços em bandejas perfuradas expostas ao ar atmosférico $\left(15 \pm 2^{\circ} \mathrm{C}\right.$ e $75 \pm 3 \%$ de U.R. $)$, eles foram resfriados em câmara frigorífica por $8 \mathrm{~h}$ a $12 \pm$ $0,5^{\circ} \mathrm{C}$, visando à desaceleração metabólica.

Para a constituição dos tratamentos, os pedaços de manga foram acondicionados em bandejas rígidas de poliestireno $(0,19 \times 0,12 \mathrm{~m})$, com capacidade para $250 \mathrm{~g}$, e revestidas com os seguintes materiais: T1 (controle): filme perfurado de polietileno de baixa densidade (PEBD) de $0,006 \mathrm{~mm}$ (foram feitas 35 perfurações com agulhas, em toda a superfície da embalagem, simulando a atmosfera convencional); T2: filme de PEBD de 0,020 mm; T3: filme de PEBD de 0,060mm; T4: filme de PEBD de 0,080mm; T5: filme de polipropileno (PP) de 0,052mm, e T6: bandeja rígida de polietileno tereftalato (PET), termicamente vedada, com capacidade para $500 \mathrm{~mL}$ e dimensões de $0,22 \times 0,12 \times 0,11 \mathrm{~m}$, com tampa do mesmo material, sem perfurações e sem o revestimento plástico. As taxas de permeabilidade dos filmes utilizados, segundo protocolo descrito por Arruda et al. (2003), são: T1: $\mathrm{TPO}_{2}\left(\mathrm{~cm}^{3} \cdot \mathrm{m}^{-2} \cdot \mathrm{dia}^{-1}\right.$ a $25^{\circ} \mathrm{C}$ e $\left.1 \mathrm{~atm}\right)$ de 18.083 , $\mathrm{TPCO}_{2}\left(\mathrm{~cm}^{3} \cdot \mathrm{m}^{-2} \cdot \mathrm{dia}^{-1}\right.$ a $25^{\circ} \mathrm{C}$ e $\left.1 \mathrm{~atm}\right)$ de $75.552 \mathrm{e}$ área de permeabilidade de $790 \mathrm{~cm}^{2} ; \mathrm{T} 2$ : $\mathrm{TPO}_{2}$ de 9.220, $\mathrm{TPCO}_{2}$ de 38.005 e área de permeabilidade de $805 \mathrm{~cm}^{2}$; T3: $\mathrm{TPO}_{2}$ de $3.565, \mathrm{TPCO}_{2}$ de 13.888 e área de permeabilidade de $920 \mathrm{~cm}^{2}$; T4: $\mathrm{TPO}_{2}$ de 2.302, $\mathrm{TPCO}_{2}$ de 8.756 e área de permeabilidade de $990 \mathrm{~cm}^{2}$; T5: $\mathrm{TPO}_{2}$ de $2.023, \mathrm{TPCO}_{2}$ de 6.345 e área de permeabilidade de $810 \mathrm{~cm}^{2}$; T6: TPO 2 de 12.892 e área de permeabilidade de $370 \mathrm{~cm}^{2}$. Os filmes utilizados para a AM passiva foram feitos na forma de sacolas termicamente seladas de $0,32 \times 0,25 \mathrm{~m}(1 / 4$ de espaço vazio).

Os tratamentos foram então acondicionados em câmara frigorífica a $10 \pm 0,5^{\circ} \mathrm{C}$ e $90 \pm 3 \%$ de U.R, por 10 dias. Durante esse período, os pedaços de mangas minimamente processadas foram analisadas nos dias $1 ; 2 ; 4 ; 6 ; 8$ e 10 de AR, quanto aos teores de sólidos solúveis (SS), determinados pela leitura refratométrica direta, em refratômetro tipo Abbe, marca 
ATAGO - N1, sendo os resultados expressos em ${ }^{\circ}$ Brix; níveis de acidez titulável (AT), determinados por titulometria de neutralização, $\mathrm{com} \mathrm{NaOH}$ a 0,01 n e ponto de viragem no $\mathrm{pH}=8,2$, sendo os resultados expressos em $\mathrm{g}$ de ácido cítrico. $100 \mathrm{~g}$ de polpa $^{-1}$; perda de massa fresca, avaliada pela pesagem das embalagens, considerando-se a massa inicial e a massa no dia da avaliação, sendo os resultados expressos em porcentagem, tendo como máximo e admitido o valor de até $8 \%$ na perda de massa fresca total; ocorrência de desordens fisiológicas, avaliada pela porcentagem dos pedaços de manga com esses sintomas, tendo como índice de aceitabilidade máximo o valor de 5\% em cada embalagem, apresentando pigmentações não características; teor de ácido ascórbico, expresso em mg por $100 \mathrm{~g}$ de polpa, determinado segundo método de Carvalho et al. (1990), que se baseia na redução do indicador 2,6 - diclorobenzenoindolfenol (DCFI) pelo ácido ascórbico; desenvolvimento de fungos, leveduras, coliformes a 35 e $45^{\circ} \mathrm{C}$, Salmonella e bactéria lácticas, segundo a metodologia proposta por Vanderzant \& Splittstoesser (1992), em obediência à Resolução RCD n ${ }^{\circ} 12$, de 2 de janeiro de 2001, da Agência Nacional de Vigilância Sanitária (ANVISA, 2001). Ao final do experimento, realizou-se o teste de preferência, utilizando-se de 15 julgadores treinados, pela escala hedônica de 5 pontos (Moraes, 1988) gostei muitíssimo (nota 5); gostei muito (nota 4); gostei (nota 3); desgostei muito (nota 2) e desgostei muitíssimo (nota 1).

Os dados foram submetidos à análise de variância, pelo teste $\mathrm{F}$, e a comparação de médias foi efetuada pelo teste de Tukey, a 5\% de probabilidade. $\mathrm{O}$ delineamento experimental empregado foi o inteiramente casualizado, seguindo esquema fatorial $6 \times 6$ (6 dias de avaliação, 6 tipos de embalagens), com três repetições de 20 amostras cada.

\section{RESULTADOS E DISCUSSÃO}

1. Composição Gasosa nas Embalagens - Na caracterização do comportamento de cada embalagem, com relação à composição gasosa interna (Figura 1), detectaram-se quatro comportamentos distintos. Os filmes de PEBD de 0,006 mm, perfurados, e de PEBD de $0,020 \mathrm{~mm}$, não interferiram significativamente nas trocas gasosas entre a atmosfera interna dessas embalagens e o ambiente externo. Portanto, não foi possível relacionar o avanço no amadurecimento e na senescência dos pedaços de manga à ação característica da AM passiva. As concentrações internas de $\mathrm{O}_{2} \mathrm{e} \mathrm{CO}_{2}$ nesses tratamentos foram de $19,47 \%$ e $23,56 \%$, e de $0,78 \%$ e $1,1 \%$, respectivamente. Essas concentrações, que pouco variaram durante o período experimental, sempre estiveram próximas das encontradas no ar atmosférico, comprovando a ineficiência técnica dessas embalagens como barreiras físicas ao $\mathrm{O}_{2}$ e $\mathrm{CO}_{2}$. Resultados semelhantes foram descritos nos trabalhos de Arruda et al. (2003) e Neves et al. (2006), onde a utilização de filmes plásticos com espessura menor que $0,020 \mathrm{~mm}$, respectivamente em melões e carambolas minimamente processadas, também não demonstrou resistência adequada à transposição dos gases $\mathrm{O}_{2}$ e $\mathrm{CO}_{2}$. Dessa maneira, pode-se atribuir as altas taxas de permeabilidade dos filmes plásticos de PEBD de $0,006 \mathrm{~mm}$, perfurados, e PEBD de $0,020 \mathrm{~mm}$, à baixa eficiência na modificação da atmosfera interna das embalagens. Como reflexo, ao final, as mangas minimamente processadas submetidas a essas embalagens não apresentaram condição de comercialização e/ou consumo.

Situação semelhante e não satisfatória também foi detectada nas mangas minimamente processadas e embaladas com filmes de PP de $0,052 \mathrm{~mm}$. Nesse caso, mesmo havendo certa restrição à passagem do $\mathrm{O}_{2}$ e $\mathrm{CO}_{2}$, as concentrações atingidas foram consideradas ineficazes para a preservação da qualidade sensorial e microbiológica dos produtos. As concentrações de $\mathrm{O}_{2}$ nessas embalagens decresceram de maneira pouco expressiva, principalmente do ponto de vista metabólico (Cantwell, 1992), até o sexto dia de $A R$, atingindo valores médios não inferiores a $17,02 \%$. Do sexto dia em diante, as concentrações de $\mathrm{O}_{2}$ apresentaram-se constantes. Quanto ao $\mathrm{CO}_{2}$, as concentrações detectadas nessas embalagens estavam entre 2,41 e 3,26\%. Diante desses resultados, consequentemente, observou-se a diminuição na vida útil das mangas minimamente processadas e embaladas em PP de 0,052 mm. Dados semelhantes foram apresentados por Arruda et al. (2003) e Neves et al. (2006), testando embalagens de PP de 0,052 e 0,022 $\mathrm{mm}$ para o acondicionamento de melões e carambolas minimamente processados.

Nos tratamentos em que as mangas foram acondicionadas em PEBD de 0,060 e $0,080 \mathrm{~mm}$, constatou-se a diminuição na concentração interna de $\mathrm{O}_{2}$ e na manutenção de níveis satisfatórios de $\mathrm{CO}_{2}$ dentro dessas embalagens (Figura 1), que, de acordo com Cantwell (1992), poderiam permitir a conservação adequada de frutos minimamente processados. Nesses tratamentos, as concentrações de $\mathrm{O}_{2}$ e $\mathrm{CO}_{2}$, ao final do experimento, atingiram valores médios de 7,56\% e 6,37\%, respectivamente, também sendo consideradas adequadas por Donazollo et al. (2003) e Brackmann et al. (2003). Contudo, devido à demora para o estabelecimento de tais concentrações (aos 10 dias), esse comportamento não pôde contribuir para o controle no desenvolvimento de 
contaminantes microbiológicos, apesar de situaremse em patamares muito aquém dos tratamentos anteriormente citados (Tabelas 2 e 3). Além do que, o retardo no estabelecimento da atmosfera gasosa ideal dentro dessas embalagens foi crucial para a não diminuição satisfatória da velocidade da senescência dos pedaços de manga embalados em PEBD de 0,060 e $0,080 \mathrm{~mm}$ (Tabela 1 e Figura 2). Assim, supõe-se que a atividade metabólica específica das mangas 'Tommy Atkins' minimamente processadas exija barreiras mais eficientes quanto à diminuição das trocas gasosas.

No entanto, no interior das embalagens PET, já nos primeiros dias do AR das mangas minimamente processadas, estabeleceu-se a concentração atmosférica ideal para vegetais submetidos ao processamento mínimo (Figura 1). Assim sendo, aos quatro dias de AR, as concentrações de $\mathrm{O}_{2}$ e $\mathrm{CO}_{2}$ estavam, respectivamente, em $6,99 \%$ e $6,07 \%$, consideradas adequadas por Cantwell (1992), Donazollo et al. (2003) e Brackmann et al. (2003), influenciando diretamente na manutenção da qualidade geral desses frutos. Diante disso, ao final do experimento, os pedaços de manga acondicionados nas embalagens PET apresentavam condições microbiológicas satisfatórias (Tabelas $2 \mathrm{e}$ 3), ausência de danos fisiológicos na epiderme e/ou polpa (Figura 2), além do melhor controle dos níveis de vitamina C (Tabela 1) e da diminuição nas perdas de massa fresca (Figura 2), proporcionando, assim, melhor potencial de comercialização. Da mesma forma, esse mesmo tratamento foi o preferido no teste de preferência (Figura 2).

2. Contagem Microbiológica-Inicialmente, é importante salientar que, em todas as amostras analisadas, os níveis de Salmonella e de coliformes a 35 e $45^{\circ} \mathrm{C}$ encontravam-se dentro dos padrões microbiológicos estabelecidos pela ANVISA(2001), ou menores que $3 \mathrm{NMP} . \mathrm{g}^{-1}$ de amostra. Resultados semelhantes foram descritos por Pereira et al. (2003), trabalhando com goiabas var. Paluma, e Bonnas et al. (2003), trabalhando com abacaxis 'Smooth Cayenne', onde a sanitificação com o $\mathrm{NaOCl}$ e a modificação atmosférica passiva contribuíram para o eficiente controle de tais contaminantes. De acordo com Bonnas et al. (2003), a presença/ ausência de coliformes também pode funcionar como parâmetro das condições higiênico-sanitárias durante o processamento de alimentos. Assim, pelos resultados obtidos, observa-se que as condições higiênico-sanitárias foram satisfatórias neste experimento. No entanto, o simples tratamento com o hipoclorito de sódio, a exemplo do que ocorreu com o experimento de Neves et al. (2006), não foi eficiente para o controle de fungos, leveduras e bactérias lácticas em alguns dos tratamentos (Tabela 2).

Desta maneira, o desenvolvimento de fungos, leveduras e/ou bactérias lácticas, nos pedaços de manga acondicionados em filmes de PEBD de 0,006 e $0,020 \mathrm{~mm}$, foi preponderante em relação aos demais tratamentos. Esses resultados foram visualizados, em geral, a partir da segunda metade do período experimental, na ordem de $10^{2}$ a $10^{3}$ (Tabelas 2 e 3), e isso, segundo os autores, pode ter sido responsável pelo desenvolvimento de aromas e sabores desagradáveis nesses tratamentos.

Níveis intermediários de contaminação foram detectados nas mangas minimamente processadas acondicionadas nos filmes de PEBD de 0,060 e $0,080 \mathrm{~mm}$. Nesses tratamentos, a presença de fungos e leveduras, no período experimental, variou entre $1,12 \times 10^{-1}$ UFC. $\mathrm{g}^{-1}$, no início do experimento, a $8,44 \times 10^{2}$ UFC.g ${ }^{-1}$, ao final dos 10 dias de $\mathrm{AR}$, não repercutindo, por sua vez, em maiores comprometimentos sensoriais ao longo do experimento (Tabela 2). Quanto ao desenvolvimento de bactérias lácticas, os níveis variaram entre $7,20 \times 10^{1} \mathrm{UFC}^{-1} \mathrm{~g}^{-1}$, logo aos 4 dias de AR, a 9,66 x $10^{2} \mathrm{UFC}^{-1} \mathrm{~g}^{-1}$, ao final do experimento, ocasionando a descaracterização sensorial desses frutos (Tabela 3). Esses sintomas podem representar, segundo Neves et al. (2004), o efeito direto da fermentação do produto, supostamente, devido às condições da atmosfera gasosa no interior das embalagens, detectadas logo a partir do $4^{\circ}$ dia de AR. Evidencia-se, então, que a estabilização tardia da concentração gasosa nessas embalagens foi diretamente responsável pelos resultados microbiológicos detectados, depreciando, assim, a qualidade dessas mangas minimamente processadas.

Contudo, os pedaços de manga acondicionados nas embalagens PET, pela adequada estabilização da atmosfera gasosa no interior dessas embalagens, não apresentaram, ou apresentaram de maneira pouco expressiva (no caso das bactérias lácticas) indícios de contaminantes microbiológicos ao longo do experimento (Tabelas 2 e 3). Citada por Pereira et al. (2003), a inibição no crescimento de fungos e leveduras, ocorrida pelo uso de embalagens plásticas, também foi verificada por Mohd-Som et al. (1994) em brócolis minimamente processados, mostrando que elevados níveis de $\mathrm{CO}_{2}$ e baixos níveis de $\mathrm{O}_{2}$ apresentam efeitos benéficos na restrição do desenvolvimento microbiano.

3. Características físico-químicas e desordens fisiológicas - Quanto à manifestação de desordens fisiológicas, não foram detectados quaisquer indícios de sintomas decorrentes do $\mathrm{AR}$ e da 
modificação atmosférica passiva, em nenhum dos tratamentos, em nenhum tempo de análise. Todos os danos visuais e sensoriais observados durante o período experimental foram decorrentes do desenvolvimento microbiano, detectado, principalmente, nas mangas minimamente processadas e submetidas à embalagem de PEBD de 0,006 , perfurado, e PEBD de $0,020 \mathrm{~mm}$.

Foram detectados incrementos nos teores de $\mathrm{SS}$, em todos os tratamentos testados, de maneira progressiva $\left(8,9^{\circ}\right.$ Brix no momento da colheita para $12,5^{\circ}$ Brix aos 10 dias de $\mathrm{AR}$ ), contudo não foram observadas diferenças significativas entre as embalagens utilizadas. Em relação à $\mathrm{AT}$, desde o início dos trabalhos $\left(1,25 \%\right.$ de ácido cítrico. $100 \mathrm{~g}$ de polpa $\left.{ }^{-1}\right)$ até o final do experimento (de $0,189 \pm 0,005 \%$ de ácido cítrico. $100 \mathrm{~g} \mathrm{de} \mathrm{polpa}^{-1}$ ), os níveis de AT apresentaram constantes decréscimos e também não apresentaram diferença estatística entre os tratamentos. Esse comportamento indica que não houve influência significativa das embalagens plásticas no metabolismo de transformação dos ácidos orgânicos e SS durante o AR. Yamashita et al. (2002), trabalhando com mangas 'Tommy Atkins', também não observaram influência da AM na manutenção dos componentes químicos dos frutos armazenados in natura. No entanto, Bonnas et al. (2003) verificaram em abacaxis minimamente processados, pelo uso de embalagens de policloreto de Vinila (PVC) e PP, a diminuição da velocidade das perdas nos níveis de $\mathrm{AT}$, refletindo na melhor qualidade ao final do experimento. Pode-se então condicionar o efeito da embalagem plástica em relação à $\mathrm{AT}$ e aos SS, ao tipo de embalagem, à taxa de permeabilidade do filme utilizado, ao grau de processamento dos frutos e às características individuais de cada espécie/cultivar.

Os maiores teores de ácido ascórbico foram detectados nos pedaços de manga acondicionados nas embalagens PET (Figura 2). Esses frutos continham, no momento da colheita, $22,12 \mathrm{mg}$ de ácido ascórbico. $100 \mathrm{~g}^{-1}$ de polpa, chegando ao final dos 10 dias de AR com teores de 9,54 $\mathrm{mg}$ de ácido ascórbico. $100 \mathrm{~g}^{-1}$ de polpa. Esses valores corresponderam, aproximadamente, a $46 \%$ do conteúdo original do fruto no momento da colheita, o que, segundo os autores, pode ser contextualizado como pertinente do ponto de vista químico e nutricional. No entanto, a diminuição nos teores de ácido ascórbico durante o AR, em todos os tratamentos testados, já era esperada, como reflexo da senescência dos frutos minimamente processados, visto que essa substância é facilmente oxidada e fotossensível. Segundo Pina et al. (2003), a estabilidade da vitamina C em alimentos é afetada por vários fatores, como calor, luz e oxigênio, fatores esses presentes durante o AR das mangas minimamente processadas aqui utilizadas. Antoniolli et al. (2000) também observaram drásticas reduções nos teores de ácido ascórbico em abacaxis minimamente processados durante o AR. Nos demais tratamentos, onde o controle da atmosfera gasosa foi parcial ou totalmente ineficiente, não foram observadas diferenças significativas entre si, apresentando perdas variando entre $96 \pm 4 \%$ do conteúdo inicial de ácido ascórbico presente nos frutos in natura. Em valores absolutos, esses teores variaram, ao final do experimento, entre 1,49 $\mathrm{mg}$ de ácido ascórbico. $100 \mathrm{~g}^{-1}$ de polpa à completa ausência de ácido ascórbico nesses frutos.

Quanto à perda de massa fresca, quantificada nas diferentes embalagens plásticas testadas, todos os tratamentos, à exceção do controle, mostraramse eficientes e atendendo aos padrões estabelecidos, ou seja, perdas menores que $8 \%$. Entretanto, vale ressaltar que essas perdas foram sempre constantes e crescentes em relação ao período de AR. Assim, como nas demais análises, os piores índices foram detectados no tratamento-controle, onde, a partir do $6^{\circ}$ dia de avaliação, as perdas de massa fresca apresentavam-se na ordem de $8,18 \%$. Ao final do experimento, essas mesmas embalagens apresentaram perdas de $12,12 \%$, refletindo, assim, a perda excessiva de turgescência e considerável exsudação de solutos, comprometendo, dessa forma, a qualidade final desse produto. As menores perdas de umidade foram observadas nas embalagens PET, onde, ao final do experimento, a perda de umidade foi de apenas $1,20 \%$, resultado esse considerado como satisfatório.

No teste de preferência (Figura 2), comprovando todos os demais resultados das análises, os frutos das embalagens PET apresentaram os melhores índices de preferência. Esse tratamento obteve unanimidade de escolha pelos julgadores, pela manutenção dos atributos qualitativos verificados durante os 10 dias de AR. A redução da aceitação em relação aos demais tratamentos, principalmente no controle e nas mangas minimamente processadas, embaladas em PEBD de 0,006, perfurados, e PEBD $0,020 \mathrm{~mm}$, deveu-se, principalmente, ao crescimento e desenvolvimento de fungos, leveduras e bactérias láticas, proporcionando, por sua vez, alteração considerável na qualidade final do produto. 
TABELA 1- Sólidos solúveis, acidez titulável e ácido Ascórbico, em mangas ‘Tommy Atkins', minimamente processadas e armazenadas por 10 dias a $10 \pm 0,5^{\circ} \mathrm{C}$ e umidade relativa de $90 \pm 3 \%$, Boa Vista-RR - 2007.

\begin{tabular}{cccccccc}
\hline \multirow{2}{*}{ Variáveis } & Tempo & \multicolumn{7}{c}{$*$ Tratamentos } \\
\cline { 2 - 7 } & (dias) & $\mathbf{T 1}$ & $\mathbf{T 2}$ & $\mathbf{T 3}$ & $\mathbf{T 4}$ & $\mathbf{T 5}$ & $\mathbf{T 6}$ \\
\hline & $\mathbf{1}$ & $8,9 \mathrm{BA}$ & $8,8 \mathrm{BA}$ & $8,9 \mathrm{Ba}$ & $8,6 \mathrm{Ba}$ & $8,7 \mathrm{Ba}$ & $8,9 \mathrm{Ba}$ \\
Sólidos Solúveis & $\mathbf{2}$ & $9,6 \mathrm{Ba}$ & $9,5 \mathrm{Ba}$ & $9,8 \mathrm{Ba}$ & $9,2 \mathrm{Bb}$ & $9,4 \mathrm{Ba}$ & $9,5 \mathrm{Ba}$ \\
( ${ }^{\circ}$ Brix) & $\mathbf{6}$ & $10,3 \mathrm{Ba}$ & $10,8 \mathrm{Ba}$ & $10,5 \mathrm{Ba}$ & $10,2 \mathrm{Ba}$ & $10,6 \mathrm{Ba}$ & $10,4 \mathrm{Ba}$ \\
& $\mathbf{8}$ & $12 \mathrm{Aa}$ & $11,1 \mathrm{Aa}$ & $11,5 \mathrm{Aa}$ & $11,3 \mathrm{Aa}$ & $11,2 \mathrm{Aa}$ & $11,1 \mathrm{Aa}$ \\
& $\mathbf{1 0}$ & $12,4 \mathrm{Aa}$ & $12,2 \mathrm{Aa}$ & $11,8 \mathrm{Aa}$ & $11,9 \mathrm{Aa}$ & $11,6 \mathrm{Ab}$ & $11,8 \mathrm{Aa}$ \\
& $\mathbf{1}$ & $1,020 \mathrm{Aa}$ & $1,019 \mathrm{Aa}$ & $1,011 \mathrm{Aa}$ & $1,026 \mathrm{Aa}$ & $1,020 \mathrm{Aa}$ & $1,017 \mathrm{Aa}$ \\
& $\mathbf{2}$ & $0,892 \mathrm{Ba}$ & $0,841 \mathrm{Ba}$ & $0,834 \mathrm{Ba}$ & $0,880 \mathrm{Ba}$ & $0,882 \mathrm{Ba}$ & $0,860 \mathrm{Ba}$ \\
Acidez Titulá- & $\mathbf{4}$ & $0,611 \mathrm{BCa}$ & $0,670 \mathrm{BCa}$ & $0,591 \mathrm{Ca}$ & $0,590 \mathrm{Ca}$ & $0,632 \mathrm{BCa}$ & $0,660 \mathrm{BCa}$ \\
vel (\% de ácido & $\mathbf{6}$ & $0,451 \mathrm{Ca}$ & $0,444 \mathrm{Ca}$ & $0,468 \mathrm{Ca}$ & $0,445 \mathrm{Ca}$ & $0,442 \mathrm{Ca}$ & $0,440 \mathrm{Ca}$ \\
cítrico) & $\mathbf{8}$ & $0,332 \mathrm{CDa}$ & $0,346 \mathrm{CDa}$ & $0,241 \mathrm{Da}$ & $0,239 \mathrm{Da}$ & $0,337 \mathrm{CDa}$ & $0,340 \mathrm{CDa}$ \\
& $\mathbf{1 0}$ & $0,184 \mathrm{Da}$ & $0,194 \mathrm{Da}$ & $0,185 \mathrm{Da}$ & $0,188 \mathrm{Da}$ & $0,193 \mathrm{Da}$ & $0,181 \mathrm{Da}$ \\
\hline Ácido Ascórbi- & $\mathbf{2}$ & $15,53 \mathrm{Bc}$ & $14,55 \mathrm{Bd}$ & $18,07 \mathrm{Bb}$ & $20,77 \mathrm{Aa}$ & $20,53 \mathrm{Aa}$ & $21,14 \mathrm{Aa}$ \\
co (mg de ác. & $\mathbf{4}$ & $7,54 \mathrm{Ccd}$ & $7,05 \mathrm{Cd}$ & $8,20 \mathrm{Cc}$ & $15,90 \mathrm{Bb}$ & $16,55 \mathrm{Bab}$ & $17,25 \mathrm{ABa}$ \\
ascórbico.100g & $\mathbf{6}$ & $4,25 \mathrm{Ce}$ & $3,33 \mathrm{Cf}$ & $5,62 \mathrm{Cd}$ & $8,36 \mathrm{Cc}$ & $9,84 \mathrm{Cb}$ & $12,40 \mathrm{Ba}$ \\
de polpa $\left.{ }^{-1}\right)$ & $\mathbf{8}$ & $2,98 \mathrm{Ce}$ & $2,54 \mathrm{Ce}$ & $3,78 \mathrm{Cd}$ & $5,12 \mathrm{Cc}$ & $7,23 \mathrm{Cb}$ & $10,12 \mathrm{CBa}$ \\
& $\mathbf{1 0}$ & $0 \mathrm{Db}$ & $0,16 \mathrm{Db}$ & $0 \mathrm{Db}$ & $1,18 \mathrm{Db}$ & $1,49 \mathrm{Db}$ & $9,54 \mathrm{Ca}$ \\
\hline
\end{tabular}

As médias seguidas das mesmas letras, nas linhas (tratamento - minúscula) e nas colunas (tempo - maiúscula), não diferem entre si, pelo teste de Tukey $(\mathrm{p} \leq 0,05)$.

D.M.S.: Diferença mínima significativa ao nível de 0,05 , C.V.: Coeficiente de variação

* T1: PEBD de 0,006mm; T2: PEBD de 0,020mm; T3: PEBD de 0,060mm; T4: PEBD de 0,080mm.; T5: PP de 0,052mm; T6: PET.

TABELA 2 - Contagem de Fungos e Leveduras, em mangas 'Tommy Atkins', minimamente processadas e armazenadas por 10 dias a $10 \pm 0,5{ }^{\circ} \mathrm{C}$ e umidade relativa de $90 \pm 3 \%$, Boa Vista-RR 2007.

\begin{tabular}{ccccccc}
\hline $\begin{array}{c}\text { Tempo } \\
\text { (dias) }\end{array}$ & T1 & T2 & T3 & T4 & T5 & T6 \\
\hline $\mathbf{1}$ & $5,21 \times 10^{1} \mathrm{Ea}^{*}$ & $2,45 \times 10^{-1} \mathrm{~Eb}$ & $5,02 \times 10^{-1} \mathrm{Cb}$ & $1,12 \times 10^{-1} \mathrm{Cb}$ & $1,21 \times 10^{-2} \mathrm{Dc}$ & $0 \mathrm{Ce}$ \\
$\mathbf{2}$ & $3,43 \times 10^{2} \mathrm{Da}$ & $2,34 \times 10^{1} \mathrm{Db}$ & $4,21 \times 10^{-1} \mathrm{Cc}$ & $3,14 \times 10^{-1} \mathrm{Cc}$ & $4,12 \times 10^{-3} \mathrm{Cd}$ & $0 \mathrm{Ce}$ \\
$\mathbf{4}$ & $7,54 \times 10^{2} \mathrm{Da}$ & $7,12 \times 10^{1} \mathrm{Db}$ & $8,15 \times 10^{-1} \mathrm{Cc}$ & $9,91 \times 10^{-1} \mathrm{Cc}$ & $9,41 \times 10^{-3} \mathrm{Cd}$ & $0 \mathrm{Ce}$ \\
$\mathbf{6}$ & $2,51 \times 10^{3} \mathrm{Ca}$ & $2,01 \times 10^{2} \mathrm{Cb}$ & $3,42 \times 10^{1} \mathrm{Bc}$ & $2,29 \times 10^{1} \mathrm{Bc}$ & $2,91 \times 10^{-2} \mathrm{Bd}$ & $0,91 \times 10^{-4} \mathrm{Be}$ \\
$\mathbf{8}$ & $5,54 \times 10^{4} \mathrm{Ba}$ & $1,08 \times 10^{3} \mathrm{Bb}$ & $1,56 \times 10^{2} \mathrm{Ac}$ & $9,42 \times 10^{1} \mathrm{Bd}$ & $6,48 \times 10^{-1} \mathrm{Ae}$ & $2,91 \times 10^{-4} \mathrm{Bf}$ \\
$\mathbf{1 0}$ & $8,74 \times 10^{6} \mathrm{Aa}$ & $4,91 \times 10^{5} \mathrm{Ab}$ & $8,44 \times 10^{2} \mathrm{Ac}$ & $8,29 \times 10^{2} \mathrm{Ac}$ & $8,89 \times 10^{-1} \mathrm{Ad}$ & $7,91 \times 10^{-3} \mathrm{Ae}$ \\
\hline
\end{tabular}

As médias seguidas das mesmas letras, nas linhas (tratamento - minúscula) e nas colunas (tempo - maiúscula), não diferem entre si, pelo teste de Tukey $(\mathrm{p} \leq 0,05)$.

*Unidades formadoras de colônia por grama de amostra (UFC. $\left.\mathrm{g}^{-1}\right)$.

** T1: PEBD de 0,006mm; T2: PEBD de 0,020mm; T3: PEBD de 0,060mm; T4: PEBD de 0,080mm.; T5: PP de 0,052mm; T6: PET. 
TABELA 3 - Contagem de Bactéria Lácticas, em mangas 'Tommy Atkins', minimamente processadas e armazenadas por 10 dias a $10 \pm 0,5{ }^{\circ} \mathrm{C}$ e umidade relativa de $90 \pm 3 \%$, Boa Vista-RR 2007.

\begin{tabular}{ccccccc}
\hline Tempo & \multicolumn{5}{c}{ **Tratamentos } \\
\cline { 2 - 7 } (dias) & T1 & T2 & T3 & T4 & T5 & T6 \\
\hline $\mathbf{1}$ & $1,25 \times 10^{-1} \mathrm{Da}$ & $4,41 \times 10^{-3} \mathrm{~Eb}$ & $2,16 \times 10^{-3} \mathrm{Cb}$ & $3,44 \times 10^{-3} \mathrm{Db}$ & $5,08 \times 10^{-4} \mathrm{Ec}$ & $0 \mathrm{Ad}$ \\
$\mathbf{2}$ & $4,10 \times 10^{2} \mathrm{Ca}$ & $2,51 \times 10^{-1} \mathrm{Db}$ & $5,21 \times 10^{-1} \mathrm{Bb}$ & $6,01 \times 10^{-1} \mathrm{Cb}$ & $5,92 \times 10^{-3} \mathrm{Dc}$ & $0 \mathrm{Ad}$ \\
$\mathbf{4}$ & $2,18 \times 10^{3} \mathrm{Ba}$ & $3,33 \times 10^{2} \mathrm{Cb}$ & $4,01 \times 10^{2} \mathrm{Ab}$ & $7,20 \times 10^{1} \mathrm{Bc}$ & $3,49 \times 10^{-3} \mathrm{Dd}$ & $0 \mathrm{Ae}$ \\
$\mathbf{6}$ & $9,01 \times 10^{3} \mathrm{Ba}$ & $5,31 \times 10^{2} \mathrm{Cb}$ & $6,49 \times 10^{2} \mathrm{Ab}$ & $4,38 \times 10^{2} \mathrm{Ab}$ & $8,19 \times 10^{-2} \mathrm{Cc}$ & $0 \mathrm{Ad}$ \\
$\mathbf{8}$ & $3,08 \times 10^{5} \mathrm{Aa}$ & $2,96 \times 10^{3} \mathrm{Bb}$ & $7,73 \times 10^{2} \mathrm{Ac}$ & $1,21 \times 10^{2} \mathrm{Ac}$ & $5,34 \times 10^{-1} \mathrm{Bd}$ & $0 \mathrm{Ae}$ \\
$\mathbf{1 0}$ & $4,22 \times 10^{5} \mathrm{Aa}$ & $1,48 \times 10^{4} \mathrm{Ab}$ & $9,66 \times 10^{2} \mathrm{Ac}$ & $8,94 \times 10^{2} \mathrm{Ac}$ & $1,42 \times 10^{1} \mathrm{Ad}$ & $0 \mathrm{Ae}$
\end{tabular}

As médias seguidas das mesmas letras, nas linhas (tratamento - minúscula) e nas colunas (tempo - maiúscula), não diferem entre si, pelo teste de Tukey $(\mathrm{p} \leq 0,05)$.

*Unidades formadoras de colônia por grama de amostra (UFC. $\left.\mathrm{g}^{-1}\right)$.

** T1: PEBD de 0,006mm; T2: PEBD de 0,020mm; T3: PEBD de 0,060mm; T4: PEBD de 0,080mm.; T5: PP de 0,052mm; T6: PET.
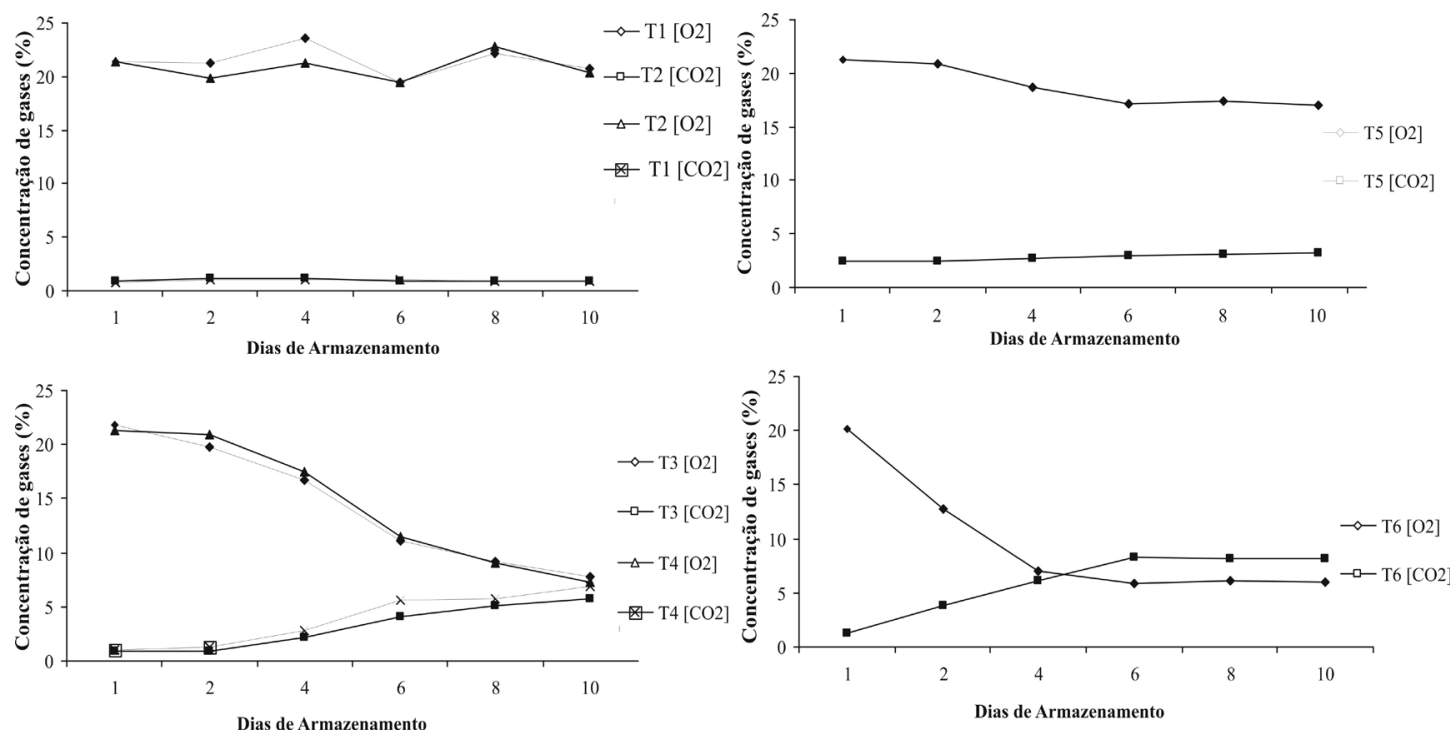

As médias seguidas das mesmas letras, nas linhas (tratamento - minúscula) e nas colunas (tempo - maiúscula), não diferem entre si, pelo teste de Tukey $(\mathrm{p} \leq 0,05)$.

** T1: PEBD de 0,006mm; T2: PEBD de 0,020mm; T3: PEBD de 0,060mm; T4: PEBD de 0,080mm.; T5: PP de 0,052mm; T6: PET.

FIGURA 1 - Composição gasosa do espaço livre das embalagens com mangas ‘Tommy Atkins’, minimamente processadas e armazenadas por 10 dias a $10 \pm 0,5^{\circ} \mathrm{C}$ e umidade relativa de $90 \pm 3 \%$, Boa Vista-RR - 2007 

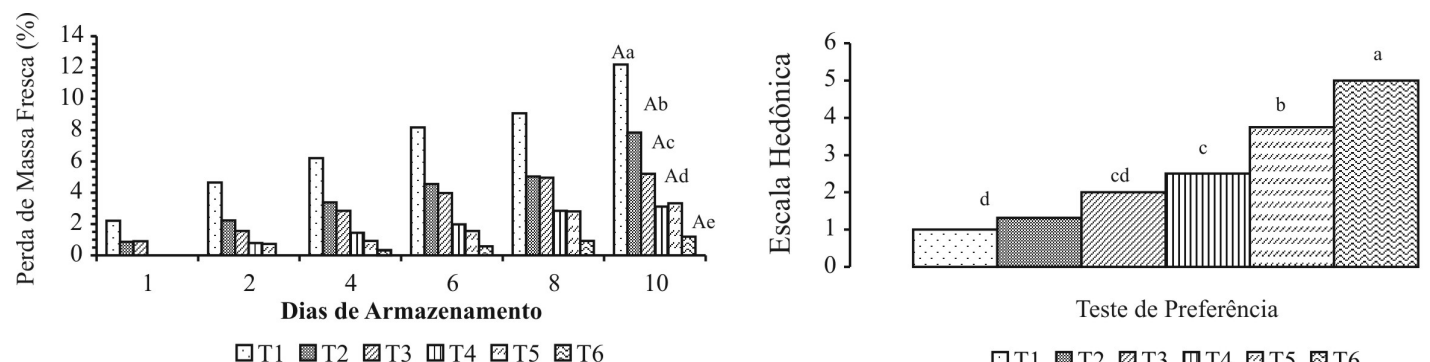

$\square \mathrm{T} 1 \square \mathrm{T} 2 \square \mathrm{T} 3 \mathrm{TT} 4 \mathrm{~T} 5 \mathrm{O} \mathrm{T} 6$

口T1 口T2 因 $\mathrm{T} 3 \mathrm{~T} 4$ 田 $\mathrm{T} 5$ 圆 $\mathrm{T} 6$

As médias seguidas das mesmas letras, nas linhas (tratamento - minúscula) e nas colunas (tempo - maiúscula), não diferem entre si, pelo teste de Tukey $(\mathrm{p} \leq 0,05)$.

* T1: PEBD de 0,006mm; T2: PEBD de 0,020mm; T3: PEBD de 0,060mm; T4: PEBD de 0,080mm.; T5: PP de 0,052mm; T6: PET.

FIGURA 2 - Perda de massa fresca e teste de preferência na polpa de mangas 'Tommy Atkins, minimamente processadas e armazenadas por 10 dias a $10 \pm 0,5^{\circ} \mathrm{C}$ e umidade relativa de $90 \pm 3 \%$, Boa Vista-RR - 2007.

\section{CONCLUSÃO}

Visando à manutenção da vida de prateleira de mangas 'Tommy Atkins' minimamente processadas, durante 10 dias de AR, recomenda-se o uso de bandejas rígidas de polietileno tereftalato (PET), com capacidade para $500 \mathrm{~mL}$, com tampa do mesmo material, sem a necessidade de revestimento plástico.

\section{REFERÊNCIAS}

ANVISA - Agência Nacional De Vigilancia Sanitária. Resolução RCD n. 12, de 2 de janeiro de 2001. Disponível em: <http://www.anvisa.gov.br.2001>. Acesso em: 29 maio 2008

AHVENAINEN, R. New approaches in improving the shelf-life of minimally processed fruit and vegetables. Trends in Food Science \& Tecnology, Amsterdam, v. 7, n. 6, p. 179-187, 1996.

ANTONIOLLI, L.R.; BENEDETTI, B.C.; CASTRO, P.R.C. Avaliação de algumas características organolépticas de frutos de abacaxizeiro (Ananas comosus L.) Meer destinados ao processamento mínimo. In: ENCONTRO DE PROCESSAMENTO MÍNIMO DE FRUTAS E HORTALIÇAS, 2.; 2000, Viçosa. Resumos... Viçosa: UFV, 2000. p.4.

ARRUDA, M.C.; JACOMINO, A.P.; SARANTÓPOULOS, C.I.G.L.; MORETTI, C.I. Qaulidade de melão minimamente processado armazenado em atmosfera modificada passiva. Horticultura Brasileira, Brasília, v.21, n.4, 2003.
BONNAS, S.D.; CHITARRA, A.B.; PRADO, M.E.T.; JÚNIOR, D.T. Qualidade do abacaxi cv. Smooth Cayenne minimamente processado. Revista Brasileira de Fruticultura, Jaboticabal, v. 25, n.2, p. 206-209, 2003.

BRACKMANN, A.; STEFFENS, C.A.; GIEHL, R.F.H. Armazenamento de pêssego 'Chimarrita' em atmosfera controlada e sob absorção de etileno. Ciência Rural, Santa Maria, v. 33, n.3,p 119-124, 2003.

CANTWELL, M. Postharvest handling systems: minimally processed fruits and vegetables. In: KADER, A.A. (Ed.). Postharvest technology of horticultural crops. Davis: University of California, 1992. p. 277-281.

CANTWELL, M.I.; SUSLOW, T.V. Postharvest handling systems: fresh-cut fruits and vegetables. In: Kader, A.A. (Ed.). Postharvest technology of horticultural crops. Davis: University of California, 2002. p.445-463.

DONAZOLLO, J.; HUNSCHE, M.; BRACKMANN, A.; WACLAWOVSKY, A.J. Utilização de filmes de polietileno de baixa densidade (PEBD) para prolongar a vida pós-colheita de morangos, cv. Oso Grande. Ciência Agrotécnica, Lavras. v.27, n.1, p.165-172, 2003.

ELESBÃO, R. A.; DURIGAN, J.F.; DONADON, J.R.; PINTO, S.A.A.; MACHADO, F.L. DE C.; BASTOS, M DO S.R. Tecnologia de processamento mínimo de manga e melão. In: SEMINÁRIO INTERNACIONAL DE PÓS-COLHEITA E PROCESSAMENTO MÍNIMO DE FRUTAS E HORTALIÇAS, 2002, Brasília-DF. Disponível em: 
$<$ http://www.cnph.embrapa.br/novidade/eventos/ semipos/texto15.pdf>. Acesso em: 29 maio 2008.

HONG, S.; KIM, D. Influence of oxygen concentration and temperature on respiratory characteristics of fresh-cut green onion. International Journal of Food Science and Technology, Oxford, v. 36, p. 283-289, 2001.

JUNQUEIRA, A.H.; LUENGO, R.F.A. Mercado diferenciados de hortaliças. Horticultura Brasileira, Brasília, v. 18, n.2, 2000.

KLUGE, R.A.; VITTI, M.C.; BASSETTO, E.; JACOMINO, A.P. Temperatura de armazenamento de tangores 'Murcote' minimamente processados. Revista Brasileira de Fruticultura, Jaboticabal, v.25, n.3, n.1, 2003.

MOHD-SOM, F.; KERBEL, E.; MARTIN, S.E.; SCHMIDT, S.J. Microflora Changes in Modified Atmosphere- Packanged Broccoli Florets Stores at Refrigerated Temperature. Journal of Food Quality, Wastport, v. 17, p. 347-360, 1994.

MORAES, M.A.C. Métodos para avaliação sensorial dos alimentos. 6.ed. Campinas: Editora Unicamp, 1988.

NEVES, L.C.; BENDER, R.J.; ROMBALDI, C.V.; VIEITES, R.L. Armazenagem em atmosfera modificada passiva de carambola-azeda (Averrhoa carambola L.) cv. 'Golden Star'. Revista Brasileira de Fruticultura, Jaboticabal, v.26, n.1,p. , 2004.
NEVES, L.C.; PRILL, M.A. de S.; SILVA, V.X. da; BENEDETTE, R.L.;VIEITES, R.L. Avaliação de diferentes tipos de atmosferas modificadas na vida útil de carambolas minimamente processadas. Revista Brasileira de Fruticultura, Jaboticabal, v.28, n.3, p.467-472, 2006

PEREIRA, L.M.; RODRIGUES, A.C.C.; SARANTÓPOULOS, C.I.G.de L. Vida de prateileira de goiabas minimamente processadas acondicionadas em embalagens sob atmosfera modificada. Ciência e Tecnologia de Alimentos, Campinas, v. 23, n.3, p. 427-433, 2003.

PINA, M.G.M.; MAIA, G.A.; FILHO, M. de S.M DE S.; FIGUEIREDO, R.W.de; MONTEIRO, J.C.S Processamento e conservação de manga por métodos combinados Revista Brasileira de Fruticultura, Jaboticabal, v. 25, n. 1, p. 63-66, 2003.

VANDERZANT, C.; SPLITTSTOESSER, D.F. Compendium of methods for the microbiological examination of foods. Washington: American Public Health Association, 1992. 600p.

VERLINDEN, B.E.; NICOLAI, B.M. Fresh-cut of fruits and vegetables. Acta Horticulturae, Wageningen, n.518, p.223-230, 2000.

YAMASHITA, F.; MIGLIORANZA, L.H. da S.; MIRANDA, L. de A.; SOUZA, C.M. de A. Effects of packaging and temperature on postharvest of atemoya. Revista Brasileira de Fruticultura, Jaboticabal, v.24, n.3, p.658-660, 2002. 